\title{
Neuroquímica da esquizofrenia: papel dos fosfolípides
}

Wagner F Gattaz

Laboratório de Neurociências (LIM-27), Departamento e Instituto de Psiquiatria da Faculdade de Medicina da USP

\section{Introdução}

Não se encontrou ainda uma alteração neuroquímica definitiva na esquizofrenia. Para a maioria de achados positivos descritos na literatura, encontram-se também estudos com resultados negativos. Em outra ocasião, já discutimos os potenciais aspectos metodológicas que vêm contribuindo para a inconsistência de resultados até o momento. ${ }^{1}$ Alguns resultados experimentais mais consistentes foram resumidos recentemente ${ }^{2}$ e incluem alterações de receptores dopaminérgicos (D2-like) nos gânglios da base, de receptores serotoninérgicos (5-HT1A e 5HT2) no córtex frontal, assim como alterações nos sistemas gabaérgicos e glutamatérgicos.

Se a esquizofrenia for considerada uma síndrome resultante de um distúrbio na maturação do cérebro, é provável que parte das alterações neuroquímicas descritas sejam secundárias à alteração básica da plasticidade cerebral durante a infância e a adolescência. Isso implica dirigir a procura de fatores causais e primários dentre os processos biológicos que modulam a maturação cerebral. Nesse sentido, a evidência experimental acumulada na última década sugere que um distúrbio no metabolismo dos fosfolípides seja um candidato ao papel de fator primário na biologia da esquizofrenia.

\section{Metabolismo dos fosfolípides e função cerebral}

Processos do metabolismo da membrana neuronal influenciam de forma decisiva a plasticidade e a função do cérebro. A camada dupla de fosfolipídeos que compõe a membrana neuronal determina as suas propriedades físico-químicas. Assim, a estrutura da membrana determina a sua fluidez, influencia o número e a afinidade dos receptores nela ligados, e modula os processos de transdução de sinais. Os fosfolípides são os principais componentes da membrana neuronal. Eles servem também como substrato para a síntese de mediadores intra e extracelulares, aumentando, assim, sua relevância para a neurotransmissão. ${ }^{3}$

O metabolismo dos fosfolípides é controlado por enzimas ligadas à membrana. A enzima fosfolipase A2 (PLA2) tem aqui uma função primordial. A PLA2 catalisa nos fosfolipídeos a hidrólise de ácidos graxos ligados à posição sn-2, liberando da membrana ácidos graxos livres e lisofosfolípides. Tanto o ácido araquidônico livre e as prostaglandinas que deles resultam, como a lisofosfatidilcolina, são importantes mediadores na transmissão e no processamento de sinais neuronais. ${ }^{4}$ Assim, alterações na atividade da PLA2 podem ser relevantes para o surgimento e para o curso de diferentes doenças neuropsiquiátricas. De fato, distúrbios da PLA2 já foram descritos em pacientes com esclerose múltipla e com epilepsia do lobo temporal.,

\section{Distúrbio do metabolismo de fosfolípides na esquizofrenia}

Diversos estudos in vivo e post mortem encontraram um distúrbio do metabolismo de fosfolípides no cérebro de pacientes esquizofrênicos. Estudos com 31P-espectroscopia magnética sugerem uma aceleração do metabolismo de fosfolipídeos no lobo frontal de esquizofrênicos. Nesses estudos investigaramse as ressonâncias dos fosfomonoesterase (PME) e dos fosfodiesterase (PDE), respectivamente, os precursores e os metabólitos dos fosfolípides no cérebro. Esquizofrênicos mostravam uma diminuição dos PME e um aumento dos PDE no lobo frontal. ${ }^{7}$ Uma aceleração do metabolismo de fosfolípides foi também encontrada em um estudo post mortem no lobo frontal de esquizofrênicos. ${ }^{8}$ Esses achados no cérebro, apoiaram resultados de estudos anteriores que encontraram na esquizofrenia uma diminuição da concentração de fosfolípides nos eritrócitos ${ }^{9}$ e, de nosso grupo, um aumento da concentração do metabólito lisofosfatidolcolina na membrana de plaquetas. ${ }^{10}$ Também a concentração do ácido araquidônico em eritrócitos foi encontrada reduzida em um subgrupo de pacientes esquizofrênicos. ${ }^{11}$

Como a PLA2 é uma enzima-chave no metabolismo dos fosfolipídeos, iniciamos as primeiras investigações de sua atividade na esquizofrenia. Em dois estudos independentes, usando um método fluorométrico, encontramos um aumento marcante da atividade da PLA2 no plasma e no soro de pacientes esquizofrênicos (sem medicamentos) em comparação com controles sadios e com pacientes psiquiátricos não-esquizofrênicos. ${ }^{12}$

As PLAs são um grupo heterogêneo de enzimas. No homem encontram-se dois grandes grupos da enzima: a) PLA2 secretória (s PLA2) e b) PLA2 citosólica (c PLA2). As s PLA2 diferenciam-se, segundo a sua origem, em PLA2 pancreática (tipo I) e PLA2 não-pancreática (tipo II). A PLA2 citosólica e a PLA2 tipo II foram encontradas em todas as células investigadas até o momento, inclusive nos neurônios. ${ }^{13,14}$ Em colaboração com o Departamento de Patologia da Universidade de Turku, Finlândia (T.J. Nevalainen) investigamos as concentrações da PLA2 tipo I e tipo II em 43 pacientes esquizofrênicos, 41 controles sadios e 32 pacientes psiquiátricos nãoesquizofrênicos. ${ }^{15,20}$ Não encontramos qualquer diferença na concentração da PLA2 pancreática entre os três grupos. En- 
tretanto, confirmando os nossos achados anteriores, a PLA2 tipo II (não-pancreática) estava significativamente elevada em esquizofrênicos em comparação com os controles sadios $(\mathrm{p}<0,05)$ e psiquiátricos $(\mathrm{p}<0,05)$. Em esquizofrênicos, as concentrações da PLA2 tipo II apresentavam correlações significativas com a intensidade dos sintomas negativos pelo escore de anergia da "Brief psychiatric rating scale" $(\mathrm{p}<0,01)$ e pelo escore total da "Negative symptoms rating scale" $(p<0,03)$.

Esses achados sobre a esquizofrenia desencadearam investigações da PLA2 por outros laboratórios. Noponen et al ${ }^{16}$ confirmaram o aumento da atividade da PLA2 plasmática em esquizofrenia; entretanto neste estudo, o aumento foi também indicado em outros pacientes psiquiátricos não-esquizofrênicos, questionando assim a especificidade de nosso achado para a esquizofrenia. Albers et $\mathrm{al}^{17}$ não encontraram diferença na atividade da enzima no plasma de esquizofrênicos comparados com controles. Como a PLA2 no plasma representa um grupo heterogêneo de enzimas, sugerimos que nos 3 laboratórios (Gattaz, Noponen e Albers), com o uso de métodos laboratoriais diferentes, detectou-se a atividade de diferentes formas moleculares da enzima, o que explicaria a aparente inconsistência dos achados. ${ }^{18}$ Essa hipótese foi investigada por Ross et al, ${ }^{19}$ que determinaram a atividade da PLA2 no soro de esquizofrênicos e controles, simultaneamente por meio dos 3 métodos laboratoriais. Validando nosso método e nossos achados, Ross et al concluem que "em linha com Gattaz et al, ${ }^{20}$ se a PLA2 no soro for medida com seu procedimento fluorométrico, encontramos uma elevação marcante de sua atividade em pacientes com esquizofrenia”.

Para verificar se os achados no plasma e no soro refletiam um aumento da atividade da PLA2 citosólica, desenvolvemos métodos laboratoriais para investigar a atividade da enzima diretamente na membrana celular. Investigamos, em plaquetas de esquizofrênicos, simultaneamente a atividade da enzima, as concentrações dos fosfolípides por ela metabolizados e a concentração de seu principal metabólito, a lisofosfatidilcolina. As plaquetas são consideradas modelos periféricos adequados de neurônios, visto que ambos têm em comum diversas propriedades de membrana (receptores, processos metabólicos e de transdução de sinais). ${ }^{21}$

Com essa estratégia, investigamos o metabolismo dos fosfolípides em 31 pacientes esquizofrênicos. ${ }^{18} \mathrm{Cada}$ esquizofrênico foi comparado com um controle sadio e com um controle psiquiátrico não-esquizofrênico, de sexo e de idade idênticos aos dos pacientes. Todos estavam ao menos por uma semana sem medicação neuroléptica. Quinze esquizofrênicos estavam no primeiro surto da doença, nunca tendo recebido psicofármacos anteriormente. Após medirmos o metabolismo basal dos fosfolípides, 20 pacientes esquizofrênicos foram tratados durante 3 semanas de forma uniforme com $10 \mathrm{mg}$ de haloperidol/dia, período em que o metabolismo foi reavaliado semanalmente.

Encontramos um aumento significativo da atividade da PLA2 em esquizofrênicos comparados com os controles sadios $(\mathrm{p}<0,01)$ e com os pacientes psiquiátricos não-esquizofrêni$\cos (\mathrm{p}<0,03)$. A mesma diferença foi encontrada quando avaliamos apenas os 15 esquizofrênicos em primeiro surto da doença. Não havia diferença na atividade da enzima entre os pacientes não-esquizofrênicos e os controles sadios. $\mathrm{O}$ tratamento com haloperidol reduziu a atividade da PLA2 para os níveis do grupo controle.

Compatível com o aumento da PLA2, encontramos nos esquizofrênicos uma redução da concentração da fosfatidilcolina (PC), substrato da PLA2 ( $\mathrm{p}<0,006)$, enquanto a de seu principal metabólito, a lisofosfatidilcolina (LPC), estava aumentada $(p<0,005)$. Após 3 semanas de terapia neuroléptica, as concentrações de PC e de LPC normalizaram-se em níveis dos controles sadios.

$\mathrm{O}$ aumento da atividade da PLA2 em plaquetas de esquizofrênicos é consistente com os achados anteriores no soro e no plasma. ${ }^{12,20}$ É pouco provável que esse aumento seja consequiência da duração da doença ou de terapias farmacológicas anteriores, visto que esse foi também apresentado nos pacientes em primeiro surto da doença, nunca tratados anteriormente. É também pouco provável que o aumento seja um artefato de manifestações clínicas inespecíficas, como agitação e ansiedade, pois não se demonstraram diferenças entre outros pacientes psiquiátricos não-esquizofrênicos e controles sadios. Isso sugere, dentro de nossa amostra, uma especificidade de nossos achados para a esquizofrenia. Entretanto, é de interesse notar que um aumento da PLA2 já foi discutido em outras doenças neuropsiquiátricas, nas quais podem ocorrer sintomas psicóticos, como na epilepsia do lobo temporal ${ }^{6}$ e na esclerose múltipla. ${ }^{5}$ Investigações futuras deverão esclarecer se nessas doenças o aumento da PLA2 está implicado na origem das manifestações psicóticas.

Nosso achado de um aumento da atividade da PLA2 em plaquetas sugere uma aceleração do metabolismo dos fosfolipídeos na membrana de esquizofrênicos. Essa hipótese é sustentada pela redução do substrato da enzima (PC) e pelo aumento de seu metabólito hidrolítico (LPC) na membrana plaquetária dos mesmos pacientes.

\section{Implicações para a esquizofrenia}

Que implicações haveria para a patofisiologia da esquizofrenia, caso nossos achados em plaquetas estivessem refletindo um aumento da PLA2 em neurônios de esquizofrênicos? Estudos com 31P-espectroscopia magnética e estudos post mortem mostraram uma aceleração do metabolismo dos PLA2 no lobo frontal de esquizofrênicos (vide introdução). Uma disfunção do lobo frontal (hipofrontalidade) foi frequientemente descrita em esquizofrenia como, por exemplo, uma lentificação da atividade psicofisiológica no EEG-topográfico e um prejuízo do rendimento neuropsicológico. ${ }^{22} \mathrm{~A}$ disfunção frontal também é discutida como correlato biológico dos sintomas negativos da esquizofrenia (empobrecimento do pensamento, embotamento afetivo e volitivo), sintomas também encontrados em pacientes neurológicos com lesões do lobo frontal. ${ }^{23}$

Weinberger ${ }^{24}$ sugere que a hipofrontalidade em esquizofrenia é o resultado de uma diminuição da atividade dopaminérgica no lobo frontal. Portanto, foi de interesse esclarecer se um aumento da PLA2, que resultaria no encontro de uma aceleração do metabolismo de fosfolípides frontal, teria uma ação direta no sistema 
dopaminérgico, resultando na hipofrontalidade em esquizofrenia.

De fato, em experimentos comportamentais com animais de laboratório, encontramos que injeções estereotáxicas de PLA2 tanto na substância nigra pars compacta ${ }^{25}$ quanto intracerebroventricular $^{26}$ resultaram numa redução da atividade dopaminérgica. Esses resultados estão em linha com experimentos in vitro, nos quais a PLA2 inibiu a ativação da adenilciclase dopamino-sensitiva ${ }^{27}$ e reduziu a afinidade dos receptores dopaminérgicos ao antagonista $[3 \mathrm{H}]$ spiperone. ${ }^{28}$ Portanto, é plausível supor que um aumento da PLA2 no lobo frontal contribui para a hipofrontalidade na esquizofrenia por uma inibição da atividade dopaminérgica. Nesse sentido, são de interesse as correlações entre PLA2 tipo II e sintomas negativos em nosso estudo, assim como entre a aceleração do metabolismo dos fosfolípideos e o déficit neuropsicológico frontal encontrado por Deicken et al. ${ }^{29}$

Investigações futuras são necessárias para esclarecer se nossos achados em plaquetas estão refletindo um aumento cerebral da PLA2, podendo assim ser usados como marcadores periféricos para a esquizofrenia (ou, mais exatamente, para um subgrupo da doença). Essa suposição é plausível, visto que a expressão e a atividade da enzima estão sob controle genético: a) a enzima PLA2 citosólica é controlada por um locus no cromossoma $1 .^{30}$ Hudson et $\mathrm{al}^{31}$ descreveram uma associação entre uma variante genética próxima ao locus da PLA2 e esquizofrenia; b) a atividade da PLA2 é controlada por um grupo de proteínas endógenas (phospholipase inhibiting proteins - PLIPs) determinadas por um locus no cromossoma 6, próximo ao locus de histocompatibilidade (HLA). Nós e outros autores descrevemos associações entre determinados HLAs e esquizofrenia. ${ }^{32,33}$ Mais recentemente, Moises et $\mathrm{al}^{34}$ encontraram evidência significativa de "linkage" com uma área distal da região HLA em esquizofrenia. Portanto, as PLIPs são um foco de interesse para esclarecer o aumento da PLA2 em esquizofrenia. Em nosso laboratório, iniciamos uma série de experimentos com a proteína S-100-beta, uma proteína candidata à função de uma PLIP.

As proteínas S-100 são uma família de proteínas ligantes do cálcio e presentes no sistema nervoso central e periférico de vertebrados. A S-100-beta, membro dessa família mais abundante no SNC, media a transdução de sinais do cálcio, e tem funções neurotróficas, gliotróficas e mitogênicas que influenciam o desenvolvimento e a manutenção do sistema nervoso. Um outro membro da família S100 (S100A10) tem ação moduladora do metabolismo dos fosfolípides por uma inibição da PLA2. Em colaboração com o Departamento de Bioquímica da Universidade Federal do Rio Grande do Sul, determinamos a concentração de S100-beta no plasma de 23 pacientes esquizofrênicos comparados com 23 controles sadios. Esquizofrênicos apresentaram uma redução significante de S100-beta, um achado que poderia se relacionar com o aumento (desinibição) da PLA2 e com um distúrbio da maturação cerebral em esquizofrenia.*

Projeto desenvolvido com apoio da Fapesp.

Processo ${ }^{\circ}$ 97/11083-0

\section{Referências}

1. Gattaz WF. Pesquisa neurobiológica da esquizofrenia: ingredientes básicos e condimentos insalubres. J Bras Psiquiatr 1996;45:449-51.

2. Deakin JWF. Outstanding issues neuropathology and neurochemistry of schizophrenia. In: Gattaz W, Häfner H, editors. Search for the causes of schizophrenia: balance of the century. Vol. IV. Steinkopff: Darmsadt; 1999. p. 279-90.

3. Farooqui AA, Hirashima Y, Horrocks LA. Brain phospholipases and their role in signal transduction. In: Bazan NG, Murphy MG, Tofano G, editors. Neurobiology of essential fatty acids. New York: Plenum Press; 1992. p. 11-26.

4. Bazan NG, Zorumski CF, Clark GD. The activation of phospholipase A2 and release of arachidonic acid and other lipid mediators at the synapse: the role of platelet activating factor. $\mathbf{J}$ Lipid Mediators 1993;6:421-7.

5. Woelk H, Peiler-Ichikawa K. Zur Aktivität der Phospholipase A2 gegenüber verschiedenen 1-Alk-1'-enyl-2-acyl- und 1-Alkyl-2acyl-verbindungen während der multiplen sklerose. J Neurol 1974;207:319-26.

6. Visioli F, Rodriguez-de-Turco EB, Kreisman NR, Bazan NG. Membrane lipid degradation is related to interictal cortical activity in a series of seizures. Metab Brain Dis 1994;9(2):161-70.

7. Hinsberger AD, Williamson PC, Carr TJ, Stanley JA, Drost DJ, Densmore M, et al. Magnetic resonance imaging volumetric and phosphorus 31 magnetic resonance spectroscopy measurements in schizophrenia. J Psychiatry Neurosci 1997;22(2):111-7.

8. Horrobin DF, Manku MS, Hilman H, Iain A, Glen M. Fatty acid levels in the brains of schizophrenics and normal controls. Biol Psychiatry 1991;30:795-805.

9. Rotrosen J, Wolkin A. Phospholipid and prostaglandin hypotheses of schizophrenia. In: Meltzer HY, editor. Psychopharmacology: the third generation of progress. New York: Raven Press; 1987. p. $759-64$.

10.Pangerl AM, Steudle A, Jaroni HW, Rüfer R, Gattaz WF. Increased platelet membrane lysophosphatidylcholine in schizophrenia. Biol Psychiatry 1991;30:837-40.

11.Peet M, Laugharne JDE, Horrobin DF, Reynolds GP. Arachidonic acid: a common link in the biology of schizophrenia? Arch Gen Psychiatry 1994;51:665-6.

12.Gattaz WF, Köllisch M, Thuren T, Virtanen JA, Kinnunen PKJ Increased plasma phospholipase-A2 activity in schizophrenic patients: reduction after neuroleptic therapy. Biol Psychiatry 1987;22:421-6.

13.Nevalainen TJ, Haapanen TJ. Distribution of pancreatic (group I) and synovial-type (group II) phospholipases A2 in human tissues. Inflammation 1993;17(4):453-64.

14.Stephenson DT, Manetta JV, White DL, Chiou XG, Cox L, Gitter $\mathrm{B}$, et al. Calcium-sensitive cytosolic phospholipase A2 (cPLA2) is expressed in human brain astrocytes. Brain Res 1994;637:97-105.

15.Maras A, Nevalainen TJ, Gattaz WF. Erhöhte Phospholipase A2Subtyp II korreliert mit Negativsymptomatik schizophrener Patienten. Fortschr Neurol Psychiatr 1994;62(2):117.

16.Noponen M, Sanfilipo M, Samanich K, Ryer H, Ko G, Angrist B, et al. Elevated PLA2 activity in schizophrenics and other psychiatric patients. Biol Psychiatry 1993;34:641-9.

17.Albers M, Meurer H, Märki F, Klotz, J. Phospholipase A2 activity in serum of neuroleptic-naive psychiatric inpatients. Pharmacopsychiatry 1993;26:4-98.

*Gattaz WF, Lara DR, Elkis H, Portela LV, Gonçalves CA, Tort AB, et al. Decresead S100-beta protein in schizophrenia: preliminary evidence. Schizophrenia Research 2000 (in press) 
18.Gattaz WF, Steudle A, Maras A. Increased platelet phospholipase A2 in schizophrenia. Schizophrenia Research 1995a;16:1-6.

19.Ross BM, Hudson C, Erlich J, Warsh JJ, Kish SJ. Increased phospholipid breakdown in schizophrenia. Arch Gen Psychiatry 1997;54:487-94.

20.Gattaz WF, Hübner CK, Nevalainen TJ, Thuren T, Kinnunen PKJ. Increased serum phospholipase A2 activity in schizophrenia: a replication study. Biol Psychiatry 1990;28:495-501.

21.Pletscher A. Blood platelets as neuronal models: use and limitations. Clin Neuropharmacol 1986;9(Suppl 4):344-6.

22.Gattaz WF, Mayer S, Ziegler P, Platz M, Gasser T. Hypofrontality on topographic EEG in schizophrenia. Eur Arch Clin Neurosci 1992;241:328-32.

23.Andreasen NC, Rezai K, Alliger R, Swayze II VW, Flaum M, Kirchner P, et al. Hypofrontality in neuroleptic-naive patients and in patients with chronic schizophrenia. Assessment with Xenon 133 Single-Photon Emission computed tomography and the Tower of London. Arch Gen Psychiatry 1992;49:943-58.

24. Weinberger DR. Implications of normal brain development for the pathogenesis of schizophrenia. Arch Gen Psychiatry 1987;44:660-9.

25.Brunner J, Gattaz WF. Intracerebroventricular injection of phospholipase A2 inhibits apomorphine-induced locomotion in rats. Psychiatry Research 1995;58:165-9.

26.Brunner J, Gattaz WF. Intracerebral injection of phospholipase A2 inhibits dopamine-mediated behavior in rats: possible implications for schizophrenia. Eur Arch Psychiatry Clin Neurosci 1995;246:13-6.
27.Anand-Srivastava MB, Johnson RA. Role of phospholipids in coupling of adenosine and dopamine receptors to striatal adenylate cyclase. J Neurochem 1981;36:819-828.

28. Oliveira CR, Duarte EP, Carvalho AP. Effect of phospholipase digestion and lysophosphatidylcholine on dopamine receptor binding. J Neurochem 1984;43:455-65.

29.Deicken RF, Merrin EL, Floyd TC, Weiner MW. Correlation between left frontal phospholipids and Wisconsin card sort test performance in schizophrenia. Schizophr Research 1995;14:177-81.

30.Clark JD, Lin LL, Kriz RW, Ramesha CS, Sultzman LA, Lin AY, et al. A novel, arachidonic acid-selective cytosolic PLA2 contains a $\mathrm{Ca} 2+$-dependent translocation domain with homology to PKC and GAP. Cell 1991;65(6):1043-51.

31.Hudson JC, Kennedy JL, Gotowiec A, King N, Gotjan K, Macciardi F, et al. Genetic variant near cytosolic phospholipase A2 associated with schizophrenia. Schizophrenia Research 1996;21:111-6.

32.Gattaz WF, Beckmann H. HLA antigens and schizophrenia. Lancet 1980;I:98-9.

33.Gattaz WF, Beckmann H, Mendlewicz J. HLA antigens and schizophrenia: a pool of two studies. Psychiatry Research 1981;5:123-8.

34. Moises HW, Yang L, Kristbjarnarson H, Wiese C, Byerley W, Macciardi F, et al. An international two-stage genome-wide search for schizophrenia susceptibility genes. Nature Genetics $1995 ; 11: 321-4$.

Correspondência: Wagner F Gattaz

Diretor do Laboratório de Neurociências (LIM-27) - Instituto de Psiquiatria do HCFMUSP Rua Dr. Ovídio Pires de Campos, s/n - CEP 05403-010 São Paulo, SP - E-mail: gattaz@usp.br 